\title{
A wall shear stress measurement technique using the thermal wakes of small heated spots
}

\author{
I Rudolph*, M Reyer, and W Nitsche \\ Institute of Aeronautics and Astronautics, Technical University Berlin, Berlin, Germany \\ The manuscript was received on 3 February 2009 and was accepted after revision for publication on 14 May 2009.
}

DOI: 10.1243/09544100JAERO518

\begin{abstract}
A new thermo-optical method for the measurement of wall shear stresses is presented. The technique exploits that the surface temperature field and the near wall flow are closely linked by correlating the thermal wakes of small heated spots with the wall shear stress. Numerical as well as experimental results are presented and different correlation and design parameters are examined. In contrast to recent works, where the thermal tuft length is used for a correlation with the wall shear stress, other parameters were found to be much better suited for a skin friction calibration. It is also shown that the new method has the unique capability to not only measure the magnitude of the wall shear stress but also its direction.
\end{abstract}

Keywords: wall shear stress measurement, infrared thermography, quantitative infrared thermography, sensor development

\section{INTRODUCTION}

The wall shear stress distribution can be used to detect a variety of phenomena, e.g. flow separation, reattachment, or transition. Therefore, in-depth information about the skin friction is of great importance and a variety of measurement techniques have been developed and tested in the last decades [1-3]. Commonly used techniques include skin friction balances, pressure probes (e.g. Preston tubes), and surface hot wires or hotfilms. However, many of these techniques require elaborate instrumentation and can interfere with the flow's boundary layer, thereby influencing the quantity to be measured. In addition to these quantitative techniques, a variety of qualitative, shear stress visualizing techniques have emerged in recent years, e.g. references [4] to [7]. These techniques take advantage of the fact that changes in the surface temperature are dependent on the near wall flow and the state of the flow (attached, separated, laminar, or turbulent). The corresponding wall shear stress is mostly visualized using infrared thermography or liquid crystals.

\footnotetext{
${ }^{*}$ Corresponding author: Institute of Aeronautics and Astronautics, Technical University Berlin, Marchstrasse 12, 10587 Berlin, Germany.

email:ilka.rudolph@ilr.tu-berlin.de
}

A new, promising technique that has been the focus of studies in recent years is the so-called 'thermal tuft technique'. Here, the surface temperature distribution around a small heated spot is used to visualize and quantify the near wall flow. Baughn et al. [8] first introduced the thermal tuft technique in 1995 and used it to detect the flow separation on a turbine blade. Their technique, using a laser beam to locally heat the surface and liquid crystals to visualize the thermal wake, was patented in 1999 [9]. In the following years, this laser-thermal-tuft-technique was successfully utilized to study and visualize different flow phenomena [10-13]. Batchelder and Moffat [14] presented a different method of creating a heated spot by using electrically heated aluminium pins flush mounted in a carrier plate. Other techniques utilize the thermal wake of a spot that is colder than its surroundings [15-18]. Hunt and Pantoya [19, 20] were the first to use the laser-thermal-tuft-technique as a quantitative tool by correlating the tuft length with the free-stream velocity. They found that the tuft length increases with increased velocity. Baughn et al. [21] used laser-induced thermal tufts to quantify low wall shear stresses and found an opposite trend. The tuft length decreased with increasing free-stream velocity and wall shear stress.

In the present study, a quantitative thermal tuft method for the measurement of wall shear stress magnitude and direction is described and first numerical 
and experimental results are presented. The thermal tufts in this investigation were produced through Joule heating of a flush-mounted copper wire and the resulting temperature distributions were detected with an infrared camera.

\section{EXPERIMENTAL AND NUMERICAL SET-UP}

The experimental investigations were carried out for turbulent flow conditions in an open wind tunnel facility at the Institute of Aeronautics and Astronautics at the TU Berlin. The set-up is illustrated in Fig. 1. The thermo-optical sensor and a skin friction balance were integrated into a flat plate at a distance of $x=300 \mathrm{~mm}(x / c=0.33)$ from the leading edge, where a trip wire ensured turbulent flow conditions, allowing for simultaneous measurements of wall shear stress and temperature distribution. The flat plate was mounted inside a test section with a cross-section of $300 \mathrm{~mm} \times 400 \mathrm{~mm}$ (width $\times$ height) at a height of $h=200 \mathrm{~mm}$. The skin friction balance, which was used for reference measurements of the wall shear stress, is a differential head model by Les Industries Fanny Inc. The floating element balance has a second, identical floating head inside the casing, allowing for a compensation of vibration, acceleration, and inclination effects. The floating head diameter is $8.6 \mathrm{~mm}$ (1 1/8 in), and the factory calibration has an accuracy of \pm 2 per cent. The wall shear stress on the sensor was varied by changing the wind tunnel speed and thus the freestream velocity. Measurements were carried out at ambient temperatures $\left(T_{\infty}=298 \mathrm{~K}\right)$ for velocities from $u_{\infty}=0-16.5 \mathrm{~m} / \mathrm{s}$, corresponding to Reynolds numbers of $R e_{x}=0-310000$ and wall shear stress values of $\tau_{\mathrm{w}} 0-0.8 \mathrm{~N} / \mathrm{m}^{2}$. The boundary layer thickness and momentum thickness at the sensor position ranged from $\delta_{99}=0-8.85 \mathrm{~mm}$ and $\delta_{\Theta}=0-0.86 \mathrm{~mm}$.

The heated spot was created through Joule heating of a copper wire with a diameter of $d=1 \mathrm{~mm}$ by applying a voltage to a power resistor (see the right-hand side of Fig. 1). The top of the copper wire was level with the surrounding structure's surface. To minimize lateral heat conduction, and to ensure a fast response of the thermal wake to changing flow conditions, the structure was made of a fabric-base laminate (Melamin) with a thermal conductivity of $\lambda=0.2 \mathrm{~W} / \mathrm{mK}$. The sensor was operated in a constant heat flux mode, and the resulting temperature distribution was observed and recorded using an infrared camera.

Numerical simulations were carried out to study various correlation and design parameters using the ANSYS CFX software package. The computational domain is illustrated in Fig. 2. The thermo-optical sensor was emulated by heating a small circular spot with
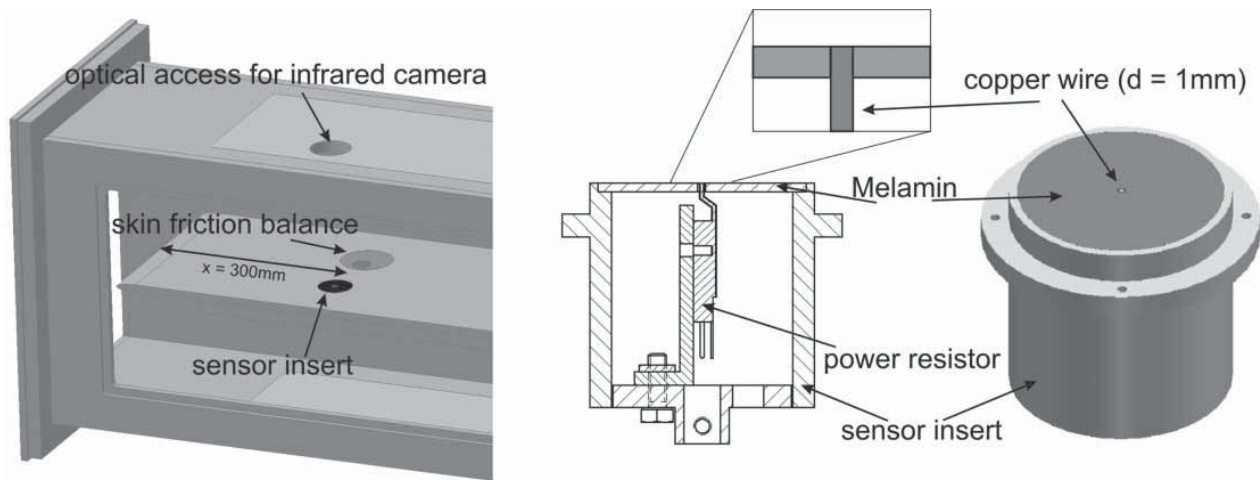

Fig. 1 Left: test section with sensor and skin friction balance. Right: sketch of the thermo-optical sensor
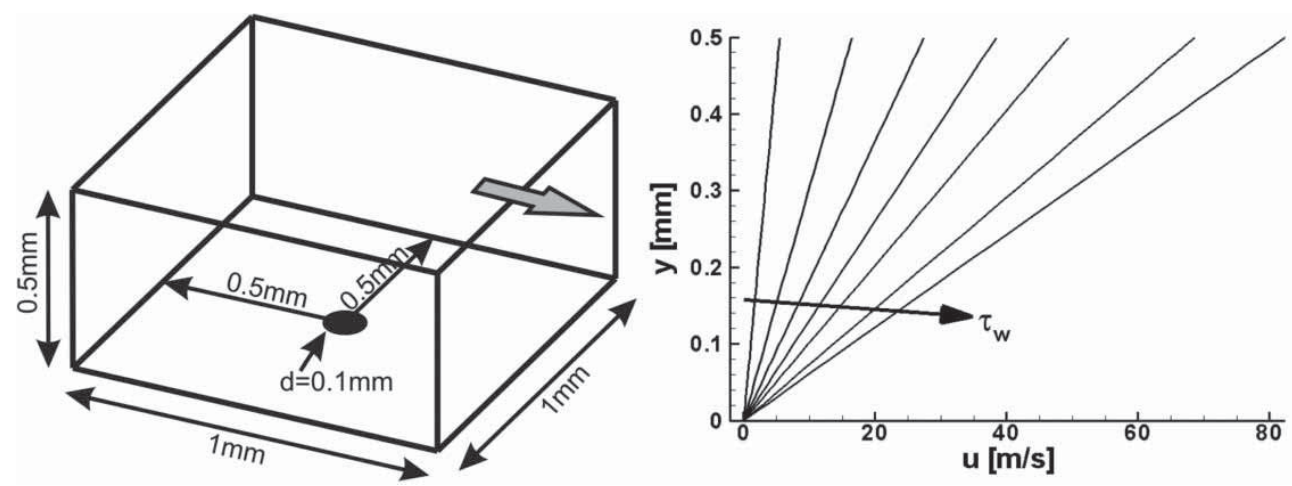

Fig. 2 Left: set-up of the numerical domain. Right: velocity profiles for various upper wall velocities 
either a constant heat flux or a constant temperature boundary condition, while the surrounding structure was kept adiabatic. The flow across the sensor was simulated using a Couette flow (see right side of Fig. 2), allowing for an easy adjustment of the wall shear stress. Since a Couette flow has a linear boundary layer profile, the wall shear stress can be calculated from the upper wall velocity

$$
\tau_{\mathrm{w}}=\mu\left(\frac{\partial u}{\partial y}\right)_{\mathrm{w}}=\mu \frac{u_{\mathrm{wall}}}{h}
$$

where $\mu$ is the dynamic viscosity, $u_{\text {wall }}$ is the upper wall velocity, and $h$ is the distance between upper and lower wall. The skin friction on the sensor was varied from $\tau_{\mathrm{w}}=0.2$ to $3.0 \mathrm{~N} / \mathrm{m}^{2}$ for laminar flow conditions and the ambient temperature was set to the same value as in the experiments $\left(T_{\infty}=298 \mathrm{~K}\right)$. The simulations were carried out for two different sensor diameters, $d=0.1$ and $1 \mathrm{~mm}$, where the larger diameter corresponds to the sensor diameter of the experiments.

The temperature boundary layers of the simulated flow cases are not contained within the viscous sublayer, i.e. $y^{+}\left(\delta_{T}\right)>5$. Therefore, the results of the Couette simulations cannot readily be transferred to the turbulent flow conditions of the experimental investigations. For this reason, a reference case of a turbulent flow across a flat plate was simulated for one shear stress value using the 'shear stress transport' (SST) turbulence model. The SST model treats the near wall region and the free-stream differently, using a $k-\omega$ model for the boundary layer and a $k-\varepsilon$ model in the free-stream region. Since the resulting temperature distribution differed only slightly from the laminar cases, it was deduced that the Couette flow could be used as a fast and computationally efficient method to investigate the possible correlation and design parameters that link the temperature distribution to the wall shear stress.

\section{NUMERICAL RESULTS}

Using the results of the numerical simulations, various correlation parameters for a skin friction calibration and different sensor modes and diameters were examined. A calibration was chosen over a Reynolds analogy approach, since it offers a more universal area of application.

\subsection{Correlation parameters}

Four correlation parameters were investigated and can be found in the schematic drawing of Fig. 3.

1. Thermal tuft length from midpoint: length between the sensor's centre and the point on a temperature isoline that is farthest away from the midpoint (distance $\overline{C E}$ in Fig. 3).

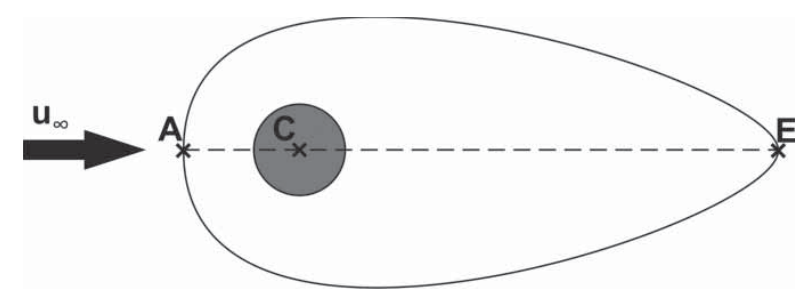

Fig. 3 Sketch of a thermal tuft temperature isoline with points used for the correlation parameters

2. Thermal tuft length overall: the longest distance between two points on a temperature isoline (distance $\overline{A E}$ in Fig. 3).

3. Area: area within or bounded by a temperature isoline, described by the number of pixels with a value larger or equal to the isoline's value.

4. Integral: area integral of the temperature distribution where only temperatures greater than the ambient temperature are taken into account, $I=$ $\iint\left(T-T_{\infty}\right) \mathrm{d} A$, where $A$ corresponds to the entire surface of the numerical domain or the field of view of the infrared camera, respectively.

The area integral is unique, because out of all four described correlation parameters it is the only one that does not need an 'evaluation temperature', i.e. a bounding temperature isoline. All other parameters need an evaluation temperature and a strong dependency of the resulting calibration on this temperature can be observed. Two different types of evaluation temperatures were studied. The first one was a fixed temperature, e.g. $T_{\text {eval }}=315 \mathrm{~K}$, the second one a variable temperature dependent on the maximum temperature, e.g. $T_{\text {eval }}=T_{\infty}+0.15\left(T_{\max }-T_{\infty}\right)$.

Figure 4 shows the temperature field around a constant heat flux sensor for four different shear stress values. The dependency of the shape of the temperature distribution on the skin friction is clearly visible. The temperature field contracts with increasing shear stress and the maximum temperature decreases. This is also mirrored in the calibration curves shown in Fig. 5 that were obtained for the same sensor, using the above-mentioned four correlation parameters and an evaluation temperature of $T_{\text {eval }}=315 \mathrm{~K}$. Since the temperature field contracts with increasing shear stress, the length and area parameters exhibit a decreasing trend. For the integral this trend is intensified by the continuously decreasing maximum temperature. This can also be observed in the resulting calibration curves shown in Fig. 5. All four parameters produce suitable calibration curves for the sensor modes and diameters investigated.

\subsection{Influence of the evaluation temperature}

The influence of the evaluation temperature on the calibration curves can be seen on the left-hand side in 

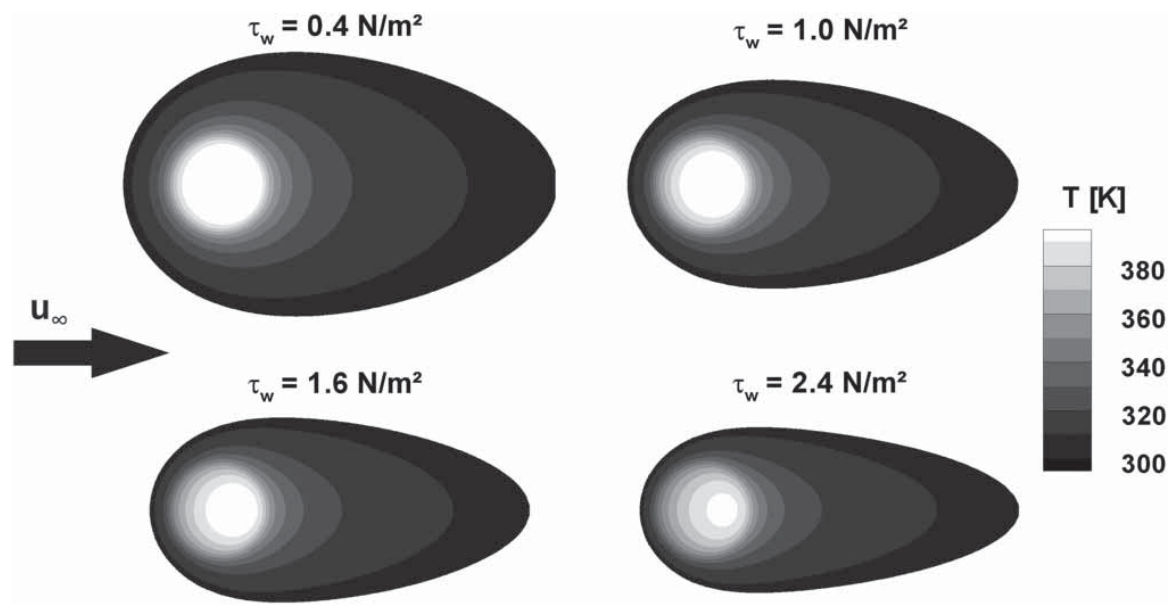

Fig. 4 Temperature field around a constant heat flux sensor for four different shear stress values
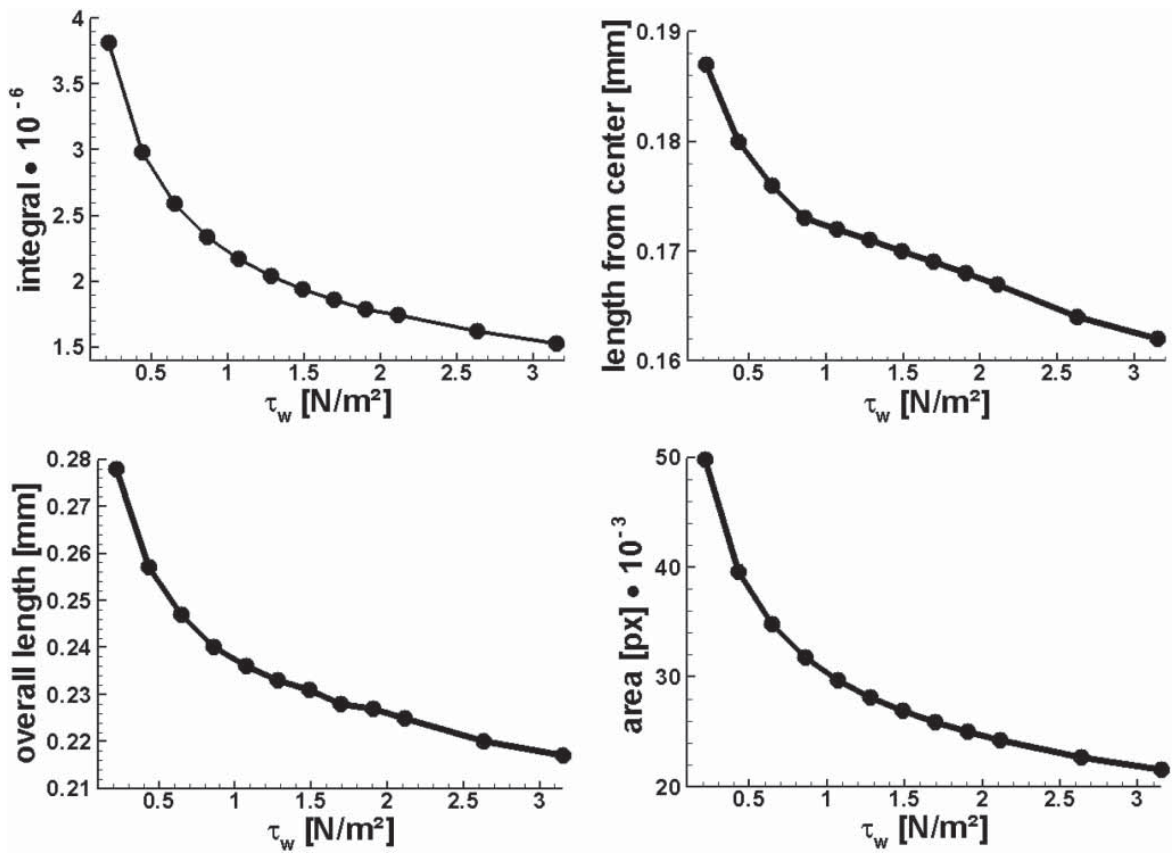

Fig. 5 Comparison of all four correlation parameters for a constant heat flux sensor $(d=0.1 \mathrm{~mm})$ and an evaluation temperature of $T_{\text {eval }}=315 \mathrm{~K}$

Fig. 6, where the tuft length calibration of a constant heat flux sensor is shown for six different evaluation temperatures. The trend of the curves reverses from decreasing to increasing tuft length when a variable temperature is chosen instead of a fixed one. This might explain the discrepancies in trends found in the literature $[\mathbf{1 9}, \mathbf{2 1}]$, where constant heat flux modes were used. This reversal in trends is due to the fact that for a constant heat flux sensor, the variable evaluation temperature decreases with increasing shear stress because the maximum temperature continuously decreases. The right-hand side of Fig. 6 shows the effect of this decreasing evaluation temperature. Here, a cross-section of a constant heat flux sensor is shown with three temperature isolines for three wall shear stress values. For a constant evaluation temperature (e.g. $T_{\text {eval }}=315 \mathrm{~K}$, black lines), the decreasing tuft length with increasing shear stress is clearly visible. However, for a decreasing evaluation temperature (i.e. light grey to grey to black), the reversal in trend is discernible as the tuft length now increases with the wall shear stress. From this cross-section, it can also be seen that the skin friction does not only influence the length but also causes the tuft to move closer to the wall as the temperature isolines contract. The reversal in trends cannot be observed for a constant temperature sensor, because here the maximum temperature is constant and independent of the skin friction, making the variable evaluation temperature in fact a constant evaluation temperature. 

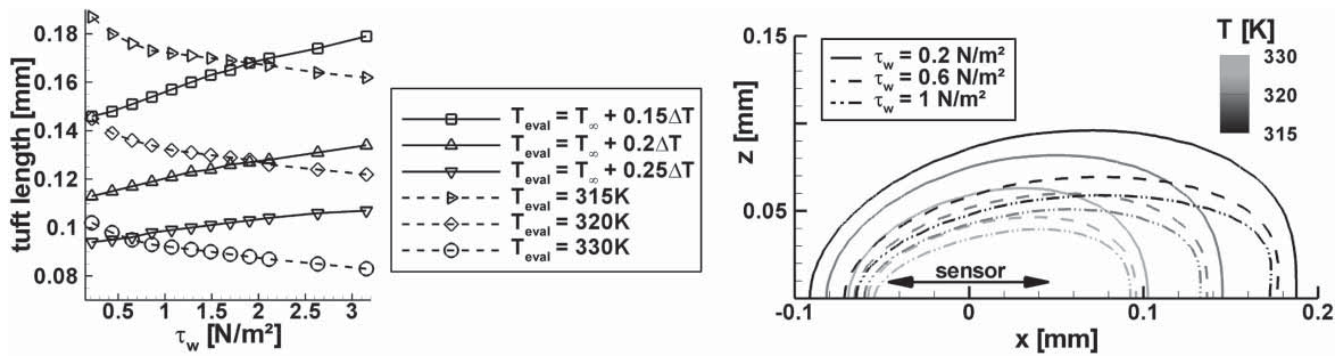

Fig. 6 Left: influence of the evaluation temperature on the calibration curves for the tuft length of a constant heat flux sensor $(d=0.1 \mathrm{~mm})$. Right: cross-section of a constant heat flux sensor with temperature isolines for three different wall shear stress values. Flow is from left to right

For the area and integral parameters the trends do not reverse, because while the decreasing evaluation temperature leads to an increasing tuft length, the tuft shape becomes more elongated and less round, leading to an overall decreasing trend for these parameters. However, the choice of evaluation temperature influences the shape of the calibration curves for both parameters. The parameters change less for a variable evaluation temperature and their calibration curves exhibit a much steeper decrease with increasing skin friction for a constant evaluation temperature.

In general, it can be said that a fixed evaluation temperature should be preferred over a variable one and lower evaluation temperatures to higher ones; however, the best choice is the integral parameter where no evaluation temperature is needed.

\subsection{Comparison of sensor modes}

The numerical simulations of the sensor were carried out for two different sensor modes. Either a constant heat flux or a constant temperature boundary condition was used to numerically heat the sensor. Figure 7 displays a comparison of the tuft length and integral calibration curves between the constant temperature and constant heat flux mode. The trend of the tuft length calibration curves reverses from decreasing with shear stress for the constant heat flux sensor to an increasing trend for the constant temperature sensor. A reversal in trend cannot be observed for the integral calibration curves; however, the steepness of the curve is affected. The constant heat flux sensor produces a steeper and therefore better suited calibration curve. The reason for this effect of the sensor mode on the shape of the calibration curves can be seen in Fig. 8. In order to keep the temperature on the sensor constant, the wall heat flux of the constant temperature sensor has to be variable and has to increase with increasing skin friction to account for the increased convective cooling (see right-hand side in Fig. 8).

This increasing heat flux leads to an increase in the tuft length for the constant temperature sensor, as can be seen in the cross-section on the left-hand side in Fig. 8. However, the temperature field still moves closer to the wall with increasing wall shear stress due to the increased near wall velocity, which explains why the trend of the integral calibration curve remains the same although the curve is less steep because the increase in tuft length adds to the integral parameter.

\subsection{Shear stress angle}

The thermo-optical sensor presented here is not only capable of measuring the wall shear stress magnitude but also its direction, because its thermal wake points in the direction of the near wall flow. The integral
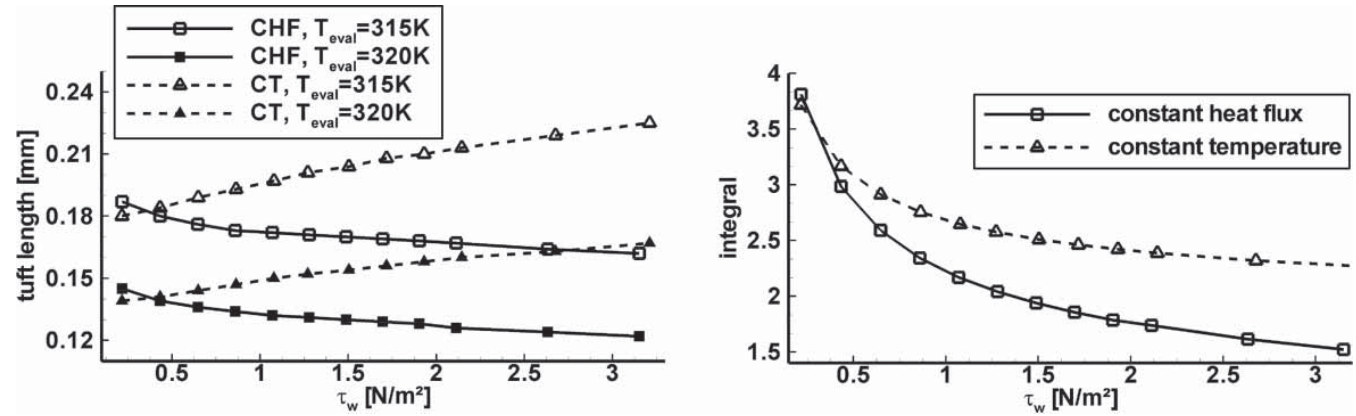

Fig. 7 Left: comparison of the tuft length calibration curves for different sensor modes and evaluation temperatures. Right: comparison of integral calibration curves for different sensor modes 

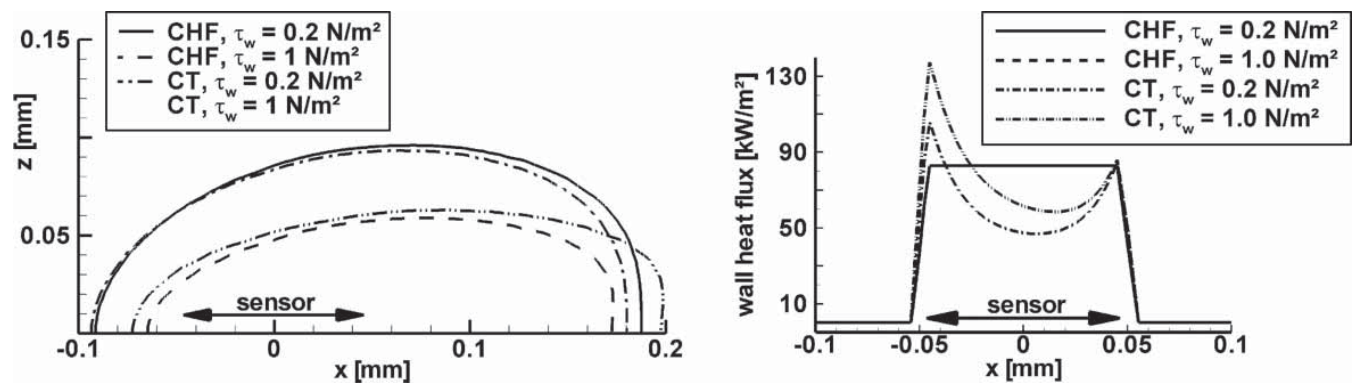

Fig. 8 Left: cross-section of a constant heat flux and a constant temperature sensor for two different shear stress values and an evaluation temperature of $T_{\text {eval }}=315 \mathrm{~K}$. Flow is from left to right. Right: comparison of the wall heat flux distribution across the sensor for a constant heat flux and a constant temperature sensor

and area parameters do not contain any information about the flow direction and thus cannot be used to determine the shear stress angle. However, the length parameters do contain directional information. The shear stress angle can be calculated from the angle between the vector pointing from the tuft midpoint to the point farthest away on the evaluation temperature's isoline ( $\overline{C E}$ in Fig. 3) and a reference direction (e.g. the $x$-axis) using

$$
\alpha=a \cos \left(\frac{\vec{a} \cdot \vec{b}}{|\vec{a}||\vec{b}|}\right)
$$

where $\vec{a}$ and $\vec{b}$ correspond to the length and the reference direction.

To study the accuracy of this method, the numerical temperature distributions were rotated with respect to the flow direction ( $x$-axis) and the angles calculated from both length parameters. For the $0^{\circ}$ case, where the temperature field was not rotated, both parameters yield the correct angles for all shear stress values. For larger angles, the tuft length from the midpoint produces slightly better results; however, both parameters are able to determine the shear stress angle with an accuracy of about $1^{\circ}$. Since the length parameters need a bounding temperature isoline, the accuracy of the angle calculation is dependent on the choice of evaluation temperature. In general, lower evaluation temperatures lead to better, more accurate results.

\subsection{Guidelines for sensor design and calibration}

The numerical simulations were carried out to investigate suitable correlation parameters and sensor design aspects. The insights gained from these investigations were applied in the experiments (see section 4). The following guidelines for a suitable sensor design and calibration were obtained:

(a) the integral parameter should be chosen for the calibration since no evaluation temperature is needed; (b) constant heat flux sensors should be preferred over constant temperature sensors since they produce steeper, more suitable calibration curves;

(c) the shear stress angle is best calculated using the tuft length from the midpoint;

(d) constant evaluation temperatures should be preferred over variable ones;

(e) larger sensor diameters lead to bigger changes in the tuft length, facilitating the calculation of the shear stress angle.

\section{EXPERIMENTAL RESULTS}

The experimental results for turbulent flow conditions and a constant heat flux sensor are presented in Figs 9 and 10. The temperature distributions for four different shear stress values, which were recorded with

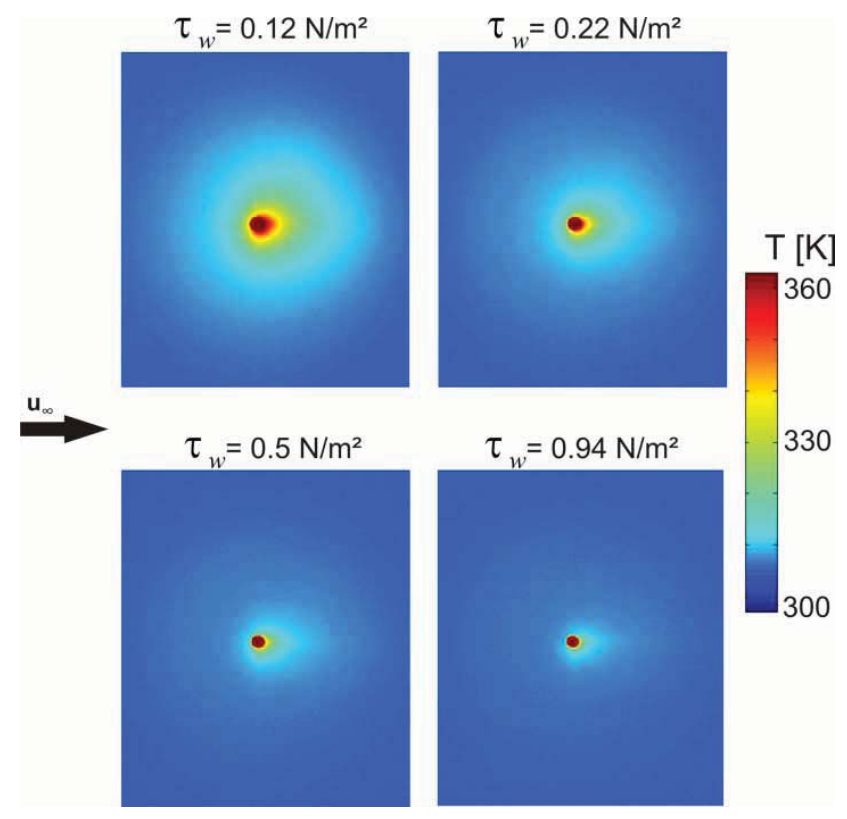

Fig. 9 Infrared images of the temperature distribution around a constant heat flux sensor for four different wall shear stress values and a sensor diameter of $d=0.1 \mathrm{~mm}$ 

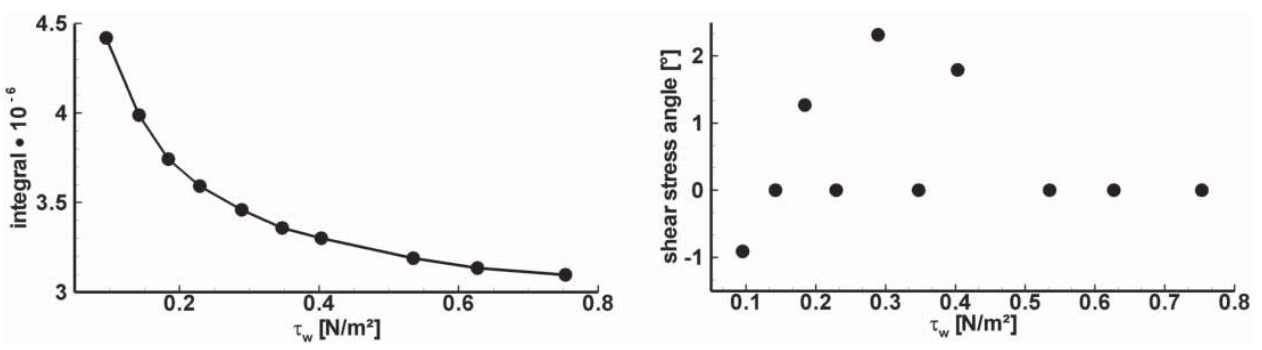

Fig. 10 Left: calibration curve for the integral parameter from the experimental results of a constant heat flux sensor and turbulent flow conditions. Right: shear stress angle calculated from the tuft length of a constant heat flux sensor for an evaluation temperature of $T_{\text {eval }}=315 \mathrm{~K}$ that was operated at a flow angle of $0^{\circ}$

an infrared camera, are displayed in Fig. 9. The trend observed in the numerical simulations is clearly confirmed in the experimental results. The temperature field contracts with increasing skin friction, leading to a decreasing tuft length.

Because of the insights from the numerical simulations, the integral parameter was used for the skin friction calibration and the tuft length from the midpoint to calculate the shear stress angle. On the lefthand side of Fig. 10, the integral calibration curve is shown. The same decreasing trend as in the numerical calibrations can be observed. The area on which the temperature field is recorded with the infrared camera is much larger than in the numerical simulations, which is the reason why the actual integral values are much larger than in the numerical calibrations. The influence of the evaluation temperature on the tuft length calibration curves could also be confirmed in the experiments. Just as in the numerical simulations, the calibration curves became less steep with increasing evaluation temperature and reversed their trend for variable evaluation temperatures.

The skin friction sensor presented here has the unique ability to not only measure the wall shear stress magnitude but also the direction of the viscous forces. The skin friction angle (with respect to a reference direction) can be calculated from the tuft length from the midpoint. In the case presented here, the sensor was not rotated with respect to the flow, so the angle should be $0^{\circ}$ for all wall shear stress values. The right-hand side in Fig. 10 displays the shear stress angles that were calculated from the infrared data. The results show some scatter, however the angle can be determined with an accuracy of about $2^{\circ}$.

\section{CONCLUSION}

A new wall shear stress measurement technique using the thermal wakes of small heated spots is presented. The technique allows for the simultaneous measurement of shear stress magnitude and direction, distinguishing it from other skin friction measurement techniques. A novel thermo-optical sensor was investigated numerically and experimentally and the results agree very well with existing literature. An agreement with reference [21] was found in the numerical as well as the experimental results, however, the opposite trend with increasing tuft length reported in reference [19] could also be verified. It could be shown that the behaviour of the tuft length with changing wall shear stress is dependent on how the temperature data are analysed and which sensor mode is used. Unlike the results of existing literature, a correlation parameter other than the tuft length was found to be best suited for a wall shear stress calibration.

Numerical simulations were carried out for laminar flow conditions and a skin friction range of $\tau_{\mathrm{w}}=0.2$ $3.0 \mathrm{~N} / \mathrm{m}^{2}$. The results were used to gain insights into suitable correlation parameters and design guidelines. Four different correlation parameters were investigated and the integral of the temperature distribution was found to be ideally suited since it does not need an evaluation temperature. However, the integral does not contain any directional information and cannot be used for the calculation of the shear stress angle. For this purpose, the tuft length from the sensor midpoint produced good results. From the numerical results, guidelines for the sensor calibration and design were deduced. The trends observed in the numerical simulations could also be confirmed in the experimental investigations, where a constant heat flux sensor was analysed for turbulent flow conditions. Overall, a very good agreement between numerical and experimental results was observed.

The thermo-optical technique presented here is well suited for measurements of wall shear stress magnitude and direction in laminar and turbulent flow conditions.

\section{REFERENCES}

1 Nitsche, W., Haberland, C., and Thuenker, R. Comparative investigations on friction drag measuring techniques in experimental aerodynamics. In Proceedings of the 14th ICAS Congress, ICAS-2.4.1, 1984, pp. 391-403. 
2 Naughton, J. and Sheplak, M. Modern developments in shear-stress measurement. Prog. Aerosp. Sci., 2002, 38, 515-570.

3 Fernholz, H. H., Janke, G., Schober, M., Wagner, P. M., and Warnack, D. New developments and applications of skin-friction measuring techniques. Meas. Sci. Technol., 1996, 7, 1396-1409.

4 Ochoa, A. D., Baughn, J. W., and Byerley, A. R. A new technique for dynamic heat transfer measurements and flow visualization using liquid crystal thermography. Int. J. Heat Fluid Flow, 2005, 26, 264-275.

5 Rudolph, I., Reyer, M., and Nitsche, W. Investigations into the visualization and quantification of wall shear stress distributions using infrared thermography and analysis of the transient data. In Proceedings of the 45th AIAA Aerospace Sciences Meeting and Exhibit, 2007, AIAA paper 2007-375.

6 Rudolph, I., Reyer, M., and Nitsche, W. Visualization of time-dependent wall shear stress distributions using infrared thermography. In Proceedings of the 12th International Symposium on Flow Visualization, ISFV12-30.3, 2006.

7 Rudolph, I., Reyer, M., and Nitsche, W. Infrared based visualization of wall shear stress distributions with a high temporal and spatial resolution. J. Vis., 2007, 10, 8.

8 Baughn, J. W., Butler, R. J., Byerley, A. R., and Rivir, R. B. An experimental investigation of heat transfer, transition and separation on turbine blades at low Reynolds numbers and high turbulence intensity. In Proceedings of the ASME International Mechanical Engineering Congress and Exposition, 1995.

9 Rivir, R. B., Baughn, J. W., Townsend, J. L., Butler, R. J., and Byerley, A. R. Thermal tuft fluid flow investigation apparatus with a color alterable thermally responsive liquid crystal layer. US Patent 5,963,292.

10 List, J., Byerley, A. R., McLaughlin, T. E., and Dyken, R. D. V. Using a plasma actuator to control laminar separation on a linear cascade turbine blade. In Proceedings of the 41st AIAA Aerospace Sciences Meeting and Exhibit, 2003.

11 Butler, R. J., Byerley, A. R., VanTreuren, K. W., and Baughn, J. W. The effect of turbulence intensity and length scale on low-pressure turbine aerodynamics. Int. J. Heat Fluid Flow, 2001, 22, 123-133.

12 Byerley, A. R., Störmer, O., Baughn, J. W., Simon, T. W., VanTreuren, K. W., and List, J. Using gurney flaps to control laminar separation on linear cascade blades. Trans. ASME, 2003, 125, 114-120.

13 Gregory, J. W. and Peterson, S. D. Flow visualization with laser-induced thermal tufts. In Proceedings of the 43rd AIAA Aerospace Sciences Meeting and Exhibit, 2005.

14 Batchelder, K. A. and Moffat R. J. Surface flow visualization using the thermal wakes of small heated spots. Expl Fluids, 1998, 25, 104-107.

15 Byerley, A. R., Störmer, O. Baugh, J. W., Simon, T. W., and VanTreuren, K. W. A cool thermal tuft for detecting surface flow direction. J. Heat Transf., 2002, 124(4), 549.
16 Baughn, J. W., Ochoa, A. D., and Smith, J. S. Surface flow visualization using encapsulated thermal tufts for steady flow and a dynamic heat transfer measurement technique for unsteady flows. J. Heat Transf., 2004, 126(4), 502.

17 Smith, J. S., Baughn, J. W., and Byerley, A. R. Surface flow visualization using thermal tufts produced by evaporatively cooled spots. Trans. ASME, 2005, 127, 186-188.

18 Smith, J. S., Baughn, J. W., and Byerley, A. R. Surface flow visualization using thermal tufts produced by an encapsulated phase change material. Int. J. Heat Fluid Flow, 2005, 26, 411-415.

19 Hunt, E. M. and Pantoya, M. L. A laser induced diagnostic technique for velocity measurements using liquid crystal thermography. Int. J. Heat Mass Transf., 2004, 47, 42854292.

20 Hunt, E. M. and Pantoya, M. L. A laser induced surface flow visualization technique using crystal thermography. In Proceedings of the 41st AIAA Aerospace Sciences Meeting and Exhibit, 2003.

21 Baughn, J. W., Byerley, A. R., and Gregory, J. W. An optical method for measuring low wall shear stresses using thermal tufts. In Proceedings of the 44th AIAA Aerospace Sciences Meeting and Exhibit, 2006, AIAA paper 2006-647.

\section{APPENDIX}

\section{Notation}

c chord length (m)

d diameter (m)

$h \quad$ height (m)

$k \quad$ turbulent kinetic energy $\left(\mathrm{m}^{2} / \mathrm{s}^{2}\right)$

$R e_{x} \quad$ Reynolds number based on $x$

$T$ temperature (K)

$T_{\text {eval }} \quad$ evaluation temperature (K)

$T_{\max } \quad$ maximum temperature (K)

$T_{\infty} \quad$ ambient temperature (K)

$u_{\text {wall }} \quad$ upper wall velocity for the Couette flow $(\mathrm{m} / \mathrm{s})$

$u_{\infty} \quad$ free-stream velocity $(\mathrm{m} / \mathrm{s})$

$x \quad$ coordinate in flow direction (m)

$y \quad$ coordinate normal to the wall (m)

$y^{+} \quad$ dimensionless wall distance

$\delta_{T} \quad$ temperature boundary layer thickness

(m)

$\delta_{\Theta} \quad$ momentum thickness (m)

$\delta_{99} \quad$ boundary layer thickness (m)

$\varepsilon \quad$ turbulent dissipation $\left(\mathrm{m}^{2} / \mathrm{s}^{3}\right)$

$\lambda$ thermal conductivity $(\mathrm{W} / \mathrm{mK})$

$\mu \quad$ dynamic viscosity $(\mathrm{kg} / \mathrm{ms})$

$\tau_{\mathrm{w}} \quad$ wall shear stress $\left(\mathrm{N} / \mathrm{m}^{2}\right)$

$\omega \quad$ turbulent frequency $(1 / \mathrm{s})$ 